International Journal of Pure and Applied Mathematics

Volume 111 No. 1 2016, 67-76

ISSN: 1311-8080 (printed version); ISSN: 1314-3395 (on-line version)

url: http://www.ijpam.eu

doi: 10.12732/ijpam.v111i1.7

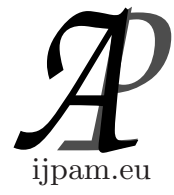

\title{
A NEW CUBICALLY CONVERGENT ITERATIVE METHOD FOR SOLVING NONLINEAR EQUATIONS
}

\author{
Young Chel Kwun ${ }^{1}$, Muhammad Saqib², Muhammad Fahad ${ }^{3}$, \\ Waqas Nazeer ${ }^{4}$, Shin Min Kang ${ }^{5}$ \\ ${ }^{1}$ Department of Mathematics \\ Dong-A University \\ Busan, 49315, KOREA \\ ${ }^{2}$ Department of Mathematics \\ Governmental Degree College \\ Kharian, 50090, PAKISTAN \\ ${ }^{3}$ Department of Mathematics \\ Bahauddin Zakariya University \\ Lahore Campus \\ Lahore, 54000, PAKISTAN \\ ${ }^{4}$ Division of Science and Technology \\ University of Education \\ Lahore, 54000, PAKISTAN \\ ${ }^{5}$ Department of Mathematics and RINS \\ Gyeongsang National University \\ Jinju, 52828, KOREA
}

\begin{abstract}
In this paper, we suggest and analyze a new third order two-step iterative method for solving nonlinear equations. To illustrate the efficiency and performance of this iterative method, we have presented comparison of newly established method with Newton's method, Abbasbandy's method, Homeier's method, Chun's method and Noor's method.
\end{abstract}

AMS Subject Classification: 47H09

Key Words: iterative methods, decomposition technique, order of convergence, nonlinear equations

Received: $\quad$ August 29, 2016

Revised: $\quad$ October 12, 2016

Published: December 2, 2016

$\S$ Correspondence author (c) 2016 Academic Publications, Ltd. url: www.acadpubl.eu 


\section{Introduction}

In recent, various techniques have been used, such as functional approximation, sampling, decomposition, geometric and Adomian approaches, to establish higher order iterative methods for solving nonlinear equations. These iterative methods consist of multi-step iterative methods. These methods are also known as predictor-corrector methods. Abbasbandy [1], Adomain [2] and Chun [4] have established some higher order one-step and two-step iterative methods by using adomain decomposition technique. They have used higher derivatives in their methods which is a serious drawback of these methods. To over come this drawback, Noor and Noor [6] established some multi-step iterative methods by applying a different decomposition technique. Initially, we do not put any restrictions on the original function $f$. In fixed point method, we rewrite $f(x)=0$ as $x=g(x)$, where:

(i) there exists $[a, b]$ such that $g(x) \in[a, b]$ for all $x \in[a, b]$,

(ii) there exists $[a, b]$ such that $\left|g^{\prime}(x)\right| \leq \lambda<1$ for all $x \in[a, b]$.

In this paper, we shall establish some algorithms using functional equation and decomposition technique given in [7]. Order of a sequence is defined as follows;

Definition 1.1. Let the sequence $\left\{x_{n}\right\}$ converges to $\alpha$. If there is a positive integer $p$ and real number $C$ such that

$$
\lim _{n \rightarrow \infty}\left|\frac{x_{n+1}-\alpha}{\left(x_{n}-\alpha\right)^{p}}\right|=C,
$$

then $p$ is order of convergence.

Theorem 1.2. (see [3]) Suppose that $\varphi \in C^{p}[a, b]$. If $\varphi^{(k)}(x)=0$ for $k=0,1,2, \ldots, m-1$ and $\varphi^{(p)}(x) \neq 0$. Then the sequence $\left\{x_{n}\right\}$ is of order $m$.

\section{Iterative Methods}

Consider the nonlinear equation

$$
f(x)=0 ; \quad x \in \mathbb{R} .
$$

We assume that $\alpha$ is a simple root of (2.1) and $\gamma$ is an initial guess sufficiently close to $\alpha$. Eqution (2.1) can be rewritten as:

$$
x=g(x),
$$




$$
x=g[\gamma+(x-\gamma)] .
$$

Using Taylors series on the right hand of above, we get

$$
x=g(\gamma)+(x-\gamma) g^{\prime}(\gamma)+G(x),
$$

where

$$
G(x)=g(x)-g(\gamma)-(x-\gamma) g^{\prime}(\gamma) .
$$

From equation (2.3) we get

$$
x=\frac{g(\gamma)-\gamma g^{\prime}(\gamma)}{1-g^{\prime}(\gamma)}+\frac{G(x)}{1-g^{\prime}(\gamma)} .
$$

It can be written in the form

$$
x=c+N(x),
$$

where

$$
c=\frac{g(\gamma)-\gamma g^{\prime}(\gamma)}{1-g^{\prime}(\gamma)}
$$

and

$$
N(x)=\frac{G(x)}{1-g^{\prime}(\gamma)} .
$$

Now, we establish a sequence of higher-order iteration schemes by applying the decomposition technique. The main idea of this technique consists in looking for a solution of Equation (2.7) having the infinite series of the form:

$$
x=\sum_{i=0}^{\infty} x_{i}
$$

The nonlinear operator $N$ can be decomposed as

$$
N\left(\sum_{i=0}^{\infty} x_{i}\right)=N\left(x_{0}\right)+\sum_{i=1}^{\infty}\left\{N\left(\sum_{j=0}^{i} x_{j}\right)\right\}
$$

which is mainly due to Noor et al. [7].

Thus we have from Equation (2.5), (2.8) and (2.9),

$$
\sum_{i=0}^{\infty} x_{i}=x_{0}+N\left(x_{0}\right)+\sum_{i=1}^{\infty}\left\{N\left(\sum_{j=0}^{i} x_{j}\right)\right\} .
$$


Thus we get the iteration schemes as

$$
\begin{aligned}
x_{0} & =c, \\
x_{1} & =N\left(x_{0}\right), \\
x_{2} & =N\left(x_{0}+x_{1}\right), \\
& \vdots \\
x_{n+1} & =N\left(x_{0}+x_{1}+\cdots+x_{n}\right), \quad n=0,1,2, \ldots .
\end{aligned}
$$

When

$$
\begin{aligned}
x & \approx x_{0} \\
& =c \\
& =\frac{g(\gamma)-\gamma g^{\prime}(\gamma)}{1-g^{\prime}(\gamma)},
\end{aligned}
$$

from above, we formulate the following algorithm:

Algorithm 2.1. For any initial value $x_{0}$, we approximate the solution $x_{n+1}$, by the iterative method:

$$
x_{n+1}=\frac{g\left(x_{n}\right)-x_{n} g^{\prime}\left(x_{n}\right)}{1-g^{\prime}\left(x_{n}\right)} \text {, }
$$

which is mainly due to Kang et al. [5] and has second order convergence.

From equation (2.4), we have

$$
\begin{aligned}
G\left(x_{0}\right) & =g\left(x_{0}\right)-g(\gamma)-\left(x_{0}-\gamma\right) g^{\prime}(\gamma) \\
& =g\left(x_{0}\right)-g(\gamma)-\left(\frac{g(\gamma)-\gamma g^{\prime}(\gamma)}{1-g^{\prime}(\gamma)}-\gamma\right) g^{\prime}(\gamma) \\
& =g\left(x_{0}\right)-g(\gamma)-\left(\frac{g(\gamma)-\gamma}{1-g^{\prime}(\gamma)}\right) g^{\prime}(\gamma) \\
& =g\left(x_{0}\right)-\left(\frac{g(\gamma)-g(\gamma) g^{\prime}(\gamma)+g(\gamma) g^{\prime}(\gamma)-\gamma g^{\prime}(\gamma)}{1-g^{\prime}(\gamma)}\right) \\
& =g\left(x_{0}\right)-\frac{g(\gamma)-\gamma g^{\prime}(\gamma)}{1-g^{\prime}(\gamma)} \\
& =g\left(x_{0}\right)-x_{0} .
\end{aligned}
$$


When

$$
\begin{aligned}
x & \approx x_{0}+x_{1} \\
& =c+N\left(x_{0}\right) \\
& =\frac{g(\gamma)-\gamma g^{\prime}(\gamma)}{1-g^{\prime}(\gamma)}+\frac{G\left(x_{0}\right)}{1-g^{\prime}(\gamma)} \\
& =\frac{g(\gamma)-\gamma g^{\prime}(\gamma)}{1-g^{\prime}(\gamma)}+\frac{g\left(x_{0}\right)-x_{0}}{1-g^{\prime}(\gamma)},
\end{aligned}
$$

from above, we formulate the algorithm as follows:

$$
x_{n+1}=\frac{g\left(x_{n}\right)-x_{n} g^{\prime}\left(x_{n}\right)}{1-g^{\prime}\left(x_{n}\right)}+\frac{g\left(\frac{g\left(x_{\mathrm{n}}\right)-x_{\mathrm{n}} g^{\prime}\left(x_{\mathrm{n}}\right)}{1-g^{\prime}\left(x_{\mathrm{n}}\right)}\right)-\frac{g\left(x_{\mathrm{n}}\right)-x_{\mathrm{n}} g^{\prime}\left(x_{\mathrm{n}}\right)}{1-g^{\prime}\left(x_{\mathrm{n}}\right)}}{1-g^{\prime}\left(x_{n}\right)},
$$

if we take

$$
y_{n}=\frac{g\left(x_{n}\right)-x_{n} g^{\prime}\left(x_{n}\right)}{1-g^{\prime}\left(x_{n}\right)}
$$

then

$$
\begin{aligned}
x_{n+1} & =y_{n}+\frac{g\left(y_{n}\right)-y_{n}}{1-g^{\prime}\left(x_{n}\right)} \\
& =\frac{g\left(y_{n}\right)-y_{n} g^{\prime}\left(x_{n}\right)}{1-g^{\prime}\left(x_{n}\right)} .
\end{aligned}
$$

Algorithm 2.2. For any initial value $x_{0}$, we approximate the solution $x_{n+1}$, by the iterative method:

Predictor step:

$$
y_{n}=\frac{g\left(x_{n}\right)-x_{n} g^{\prime}\left(x_{n}\right)}{1-g^{\prime}\left(x_{n}\right)}
$$

Corrector step:

$$
x_{n+1}=\frac{g\left(y_{n}\right)-y_{n} g^{\prime}\left(x_{n}\right)}{1-g^{\prime}\left(x_{n}\right)} .
$$

\section{Convergence Analysis}

Now we discuss the convergence analysis of Algorithm 2.2.

Theorem 3.1. Let $J \subseteq \mathbb{R}$ be an open interval and $f: J \rightarrow \mathbb{R}$ be a function and consider that the nonlinear equation $f(x)=0($ or $x=g(x)$ ) has simple root $\alpha \in J$, where $g(x): J \rightarrow \mathbb{R}$ be sufficiently smooth in the neighbourhood of the root $\alpha$. If $x_{0}$ is sufficiently close to $\alpha$, then convergence order of Algorithm 2.2 is at least 3. 
Proof. Let $\alpha$ be simple zero of $f(x)=0$ and $x=g(x)$ be its functional equation. Let errors at $n$th and $(n+1)$ th iterations be $e_{n}$ and $e_{n+1}$, respectively. Then using Taylor's expansion about $\alpha$, we have

$$
\begin{array}{r}
g\left(x_{n}\right)=\alpha+e_{n} g^{\prime}(\alpha)+\frac{1}{2} e_{n}^{2} g^{\prime \prime}(\alpha)+\frac{1}{6} e_{n}^{3} g^{\prime \prime \prime}(\alpha)+O\left(e_{n}^{4}\right), \\
g^{\prime}\left(x_{n}\right)=\alpha g^{\prime}(\alpha)+e_{n} g^{\prime \prime}(\alpha)+\frac{1}{2} e_{n}^{2} g^{\prime \prime \prime}(\alpha)+\frac{1}{6} e_{n}^{3} g^{(4)}(\alpha)+O\left(e_{n}^{4}\right), \\
g\left(x_{n}\right)-x_{n} g^{\prime}\left(x_{n}\right)=\alpha-\alpha g^{\prime}(\alpha)-\frac{1}{2}\left(g^{\prime \prime}(\alpha)+g^{\prime \prime \prime}(\alpha)\right) e_{n}^{2} \\
-\frac{1}{6}\left(2 g^{\prime \prime \prime}(\alpha)+\alpha g^{(4)}(\alpha)\right) e_{n}^{3}+O\left(e_{n}^{4}\right) .
\end{array}
$$

By substituting values in $y_{n}$ and after simplifying, we get

$$
\begin{aligned}
y_{n}= & \frac{g\left(x_{n}\right)-x_{n} g^{\prime}\left(x_{n}\right)}{1-g^{\prime}\left(x_{n}\right)} \\
= & \alpha-\frac{g^{\prime \prime}(\alpha)}{2\left(1-g^{\prime}(\alpha)\right)} e_{n}^{2}-\frac{2 g^{\prime \prime \prime}(\alpha)-2 g^{\prime \prime \prime}(\alpha) g^{\prime}(\alpha)+3\left(g^{\prime \prime \prime}(\alpha)\right)^{2}}{6\left(1-g^{\prime}(\alpha)\right)^{2}} e_{n}^{3} \\
& +O\left(e_{n}^{4}\right) . \\
g\left(y_{n}\right)= & \alpha-\frac{g^{\prime}(\alpha) g^{\prime \prime}(\alpha)}{2\left(1-g^{\prime}(\alpha)\right)} e_{n}^{2} \\
& \quad-\frac{g^{\prime}(\alpha)\left(2 g^{\prime \prime \prime}(\alpha)-2 g^{\prime \prime \prime}(\alpha) g^{\prime}(\alpha)+3\left(g^{\prime \prime \prime}(\alpha)\right)^{2}\right)}{6\left(1-g^{\prime}(\alpha)\right)^{2}} e_{n}^{3}+O\left(e_{n}^{4}\right) .
\end{aligned}
$$

Now

$$
x_{n+1}=\frac{g\left(y_{n}\right)-y_{n} g^{\prime}\left(x_{n}\right)}{1-g^{\prime}\left(x_{n}\right)} .
$$

By substituting values in above and simplifying, we have

$$
x_{n+1}=\alpha+\frac{1}{2} \frac{\left(g^{\prime \prime}(\alpha)\right)^{2}}{\left(1-g^{\prime}(\alpha)\right)^{2}} e_{n}^{3}+O\left(e_{n}^{4}\right) \text {. }
$$

Hence Algorithm 2.2 has third order convergence. 


\section{Applications}

Now we present some example to illustrate the efficiency and performance of newly devolved method namely, Algorithm 2.2. We make a comparison of Newton's method (NM), Abbasbandy's method (AM), Homeier's method (HM), Chun's method (CM), Noor's method (NR) and Algorithm 2.2 (NA) devolved in this paper (see Tables 1-7). We use $\epsilon=10^{-15}$. The following criteria are used for computer programs:

(i) $\left|x_{n}-x_{n-1}\right|<\epsilon$,

(ii) $\left|f\left(x_{n}\right)\right|<\epsilon$.

The examples are same as in Chun [4] and Noor et al. [7].

$$
\begin{aligned}
& f(x)=\sin ^{2} x-x^{2}+1, \quad g(x)=\sin x+\frac{1}{x+\sin x}, \\
& f(x)=x^{2}-e^{x}-3 x+2, \quad g(x)=\frac{e^{x}-2}{x-3}, \\
& f(x)=\cos x-x, \quad g(x)=\cos x \\
& f(x)=(x-1)^{3}-1, \quad g(x)=1+\sqrt{\frac{1}{x-1}}, \\
& f(x)=x^{3}-10, \quad g(x)=\sqrt{\frac{10}{x}}, \\
& f(x)=x e^{x^{2}}-\sin ^{2} x+3 \cos x+5, \quad g(x)=e^{-x^{2}}\left(\sin ^{2} x+3 \cos x+5\right), \\
& f(x)=e^{x^{2}+7 x-30}-1, \quad g(x)=\frac{1}{7}\left(30-x^{2}\right) .
\end{aligned}
$$

Table 1. Comparison of NM, AM, HM, CM, NR and NA

$$
\left(f(x)=\sin ^{2} x-x^{2}+1, g(x)=\sin x+\frac{1}{x+\sin x}, x_{0}=-1\right)
$$

\begin{tabular}{|c|c|c|c|}
\hline Method & Number of iterations & $x_{n}$ & $f\left(x_{n}\right)$ \\
\hline NM & 7 & 1.404491648315341226350868177 & $-1.04 \mathrm{e}-50$ \\
\hline AM & 5 & 1.404491648315341226350868177 & $-5.81 \mathrm{e}-55$ \\
\hline HM & 4 & 1.404491648315341226350868178 & $-5.4 \mathrm{e}-62$ \\
\hline CM & 5 & 1.404491648315341226350868178 & $-2.0 \mathrm{e}-63$ \\
\hline NR & 5 & 1.404491648315341226350868176 & $1.2 \mathrm{e}-28$ \\
\hline NA & 3 & 1.404491648315341226350868176 & $6.08 \mathrm{e}-28$ \\
\hline
\end{tabular}


Table 2. Comparison of NM, AM, HM, CM, NR and NA $\left(f(x)=x^{2}-e^{x}-3 x+2, g(x)=\frac{e^{\mathrm{x}}-2}{x-3}, x_{0}=2\right)$

\begin{tabular}{|c|c|c|c|}
\hline Method & Number of iterations & $x_{n}$ & $f\left(x_{n}\right)$ \\
\hline NM & 6 & 0.257530285439860760455367303 & $2.93 \mathrm{e}-55$ \\
\hline AM & 5 & 0.257530285439860760455367304 & $1.0 \mathrm{e}-63$ \\
\hline HM & 5 & 0.257530285439860760455367305 & 0 \\
\hline CM & 4 & 0.257530285439860760455367304 & $1.0 \mathrm{e}-63$ \\
\hline NR & 5 & 0.257530285439860760455367306 & $-6.0 \mathrm{e}-24$ \\
\hline NA & 5 & 0.257530285439860760455367302 & $1.63 \mathrm{e}-28$ \\
\hline
\end{tabular}

Table 3. Comparison of NM, AM, HM, CM, NR and NA $\left(f(x)=\cos x-x, g(x)=\cos x, x_{0}=1.7\right)$

\begin{tabular}{|c|c|c|c|}
\hline Method & Number of iterations & $x_{n}$ & $f\left(x_{n}\right)$ \\
\hline NM & 5 & 0.739085133215160641655372084 & $-2.03 \mathrm{e}-32$ \\
\hline AM & 4 & 0.739085133215160641655372085 & $-7.14 \mathrm{e}-47$ \\
\hline HM & 4 & 0.739085133215160641655372086 & $-5.02 \mathrm{e}-59$ \\
\hline CM & 4 & 0.739085133215160641655372087 & 0 \\
\hline NR & 4 & 0.739085133215160641655372089 & $1.4 \mathrm{e}-15$ \\
\hline NA & 4 & 0.739085133215160641655312088 & $8.74 \mathrm{e}-73$ \\
\hline
\end{tabular}

Table 4. Comparison of NM, AM, HM, CM, NR and NA

$$
\left(f(x)=(x-1)^{3}-1, g(x)=1+\sqrt{\frac{1}{x-1}}, x_{0}=3.5\right)
$$

\begin{tabular}{|c|c|c|c|}
\hline Method & Number of iterations & $x_{n}$ & $f\left(x_{n}\right)$ \\
\hline NM & 8 & 2.000000000000000000000000023 & $2.06 \mathrm{e}-42$ \\
\hline AM & 5 & 2 & 0 \\
\hline HM & 5 & 2 & 0 \\
\hline CM & 5 & 2 & 0 \\
\hline NR & 5 & 2.000000000000000000000000058 & $1.03 \mathrm{e}-23$ \\
\hline NA & 4 & 2.000000000000000000000000002 & $4.85 \mathrm{e}-45$ \\
\hline
\end{tabular}

Table 5. Comparison of NM, AM, HM, CM, NR and NA

$$
\left(f(x)=x^{3}-10, g(x)=\sqrt{\frac{10}{x}}, x_{0}=1.5\right)
$$

\begin{tabular}{|c|c|c|c|}
\hline Method & Number of iterations & $x_{n}$ & $f\left(x_{n}\right)$ \\
\hline NM & 7 & 2.154434690031883721759235664 & $2.06 \mathrm{e}-54$ \\
\hline AM & 5 & 2.154434690031883721759235667 & $-5.0 \mathrm{e}-63$ \\
\hline HM & 5 & 2.154434690031883721759235667 & $-5.0 \mathrm{e}-63$ \\
\hline CM & 5 & 2.154434690031883721759235667 & $-5.0 \mathrm{e}-63$ \\
\hline NR & 5 & 2.154434690031883721759235663 & $3.1 \mathrm{e}-42$ \\
\hline NA & 4 & 2.154434690031883721759235665 & $2.55 \mathrm{e}-70$ \\
\hline
\end{tabular}


Table 6. Comparison of NM, AM, HM, CM, NR and NA

$$
\begin{aligned}
& \left(f(x)=x e^{x^{2}}-\sin ^{2} x+3 \cos x+5\right. \\
& \left.g(x)=e^{-x^{2}}\left(\sin ^{2} x+3 \cos x+5\right), x_{0}=-2\right)
\end{aligned}
$$

\begin{tabular}{|c|c|c|c|}
\hline Method & Number of iterations & $x_{n}$ & $f\left(x_{n}\right)$ \\
\hline NM & 9 & -1.2076478271309189270094167585 & $-2.27 \mathrm{e}-40$ \\
\hline AM & 6 & -1.2076478271309189270094167584 & $-4.0 \mathrm{e}-63$ \\
\hline HM & 6 & -1.2076478271309189270094167584 & $-4.0 \mathrm{e}-63$ \\
\hline CM & 6 & -1.2076478271309189270094167584 & $-4.0 \mathrm{e}-63$ \\
\hline NR & 5 & -1.2076478271309189270094167583 & $-3.0 \mathrm{e}-31$ \\
\hline NA & 4 & -1.2076478271309189270094167582 & $-1.3 \mathrm{e}-26$ \\
\hline
\end{tabular}

Table 7. Comparison of NM, AM, HM, CM, NR and NA

$$
\left(f(x)=e^{x^{2}+7 x-30}-1, g(x)=\frac{1}{7}\left(30-x^{2}\right), x_{0}=3.5\right)
$$

\begin{tabular}{|c|c|c|c|}
\hline Method & Number of iterations & $x_{n}$ & $f\left(x_{n}\right)$ \\
\hline NM & 13 & 3.000000000000000000006 & $1.52 \mathrm{e}-47$ \\
\hline AM & 7 & 3.000000000000000000007 & $-4.33 \mathrm{e}-48$ \\
\hline HM & 8 & 3.000000000000000000001 & $2.0 \mathrm{e}-62$ \\
\hline CM & 8 & 3.000000000000000000001 & $2.0 \mathrm{e}-62$ \\
\hline NR & 7 & 3.000000000000000000011 & $-2.1 \mathrm{e}-29$ \\
\hline NA & 3 & 3.000000000000000000009 & $1.93 \mathrm{e}-33$ \\
\hline
\end{tabular}

\section{Conclusions}

In this paper, we have suggested a two-step iterative method for solving nonlinear equations. Derivation technique of this method is very simple as compared with the adomain decomposition method. It is clear from above examples that our two-step iterative method is better than the fourth-order of Chun [4] and it also performs better than the method presented in [7]. Noor et al. [7] has established free of second derivatives three-step iterative method with cubic convergence. The method established in this paper, is also free of second derivatives and is a two-step iterative method of third order convergence. This method can be considered as an improvement and refinement of previously existing method of third order convergence.

\section{Acknowledgment}

This work was supported by the Dong-A University research fund. 


\section{References}

[1] S. Abbasbandy, Improving Newton-Raphson method for nonlinear equations by modified Adomian decomposition method, Appl. Math. Comput., 145 (2003), 887-893, doi: 10.1016/S0096-3003(03)00282-0.

[2] G. Adomain, Nonlinear Atochastic Systems and Applications to Physics, Kluwer Academy Publishers. Dordrecht, 1989.

[3] E. Babolian, J. Biazar, On the order of convergence of Adomain method, Appl. Math. Comput., 130 (2002), 383-387, doi: 10.1016/S0096-3003(01)00103-5.

[4] Chun C. Iterative methods improving Newton's method by decomposition method, Comput. Math. Appl., 50 (2005), 1559-1568, doi: 10.1016/j.camwa.2005.08.022.

[5] S.M. Kang, A. Rafiq, Y.C. Kwun, A new second order iteration method for solving nonlinear equations, Abstr. Appl. Anal., 2013 (2013), Article ID 487062, 4 pages, doi: $10.1155 / 2013 / 487062$.

[6] M.A. Noor, K.I. Noor, Three-step iterative methods for nonlinear equations, Appl. math. Comput., 183 (2006), 322-327, doi: 10.1016/j.amc.2006.05.055.

[7] M.A. Noor, K.I. Noor, S.T. Mohyud-Din, A. Shabbir, An iterative method with cubic convergence for nonlinear equations, Appl. math. Comput., 183 (2006), 1249-1255, doi: 10.1016/j.amc.2006.05.133. 